\title{
Book Review on: Radioguided Surgery: Current Applications and Innovative Directions in Clinical Practice. Ken Hermann, Omgo E. Neiweg and Stephen P. Povoski (eds). Springer, Heidelberg, Germany
}

\author{
Andrea Skanjeti $^{1,2}$ - Domenico Rubello ${ }^{3}$ - Francesco Giammarile Gr, $^{1,2}$ \\ Received: 20 April 2016/Accepted: 21 April 2016/Published online: 16 May 2016 \\ (C) Springer-Verlag Berlin Heidelberg 2016
}

This theoretical and practical guide was edited by Ken Hermann (a distinguished nuclear physician), Omgo E. Neiweg and Stephen P. Povoski (two eminent surgeons), in collaboration of many international experts. All the authors who have contributed to this publication are well-known experts in the very intriguing field of radioguided surgery.

The declared aim of the authors is to provide an original and exhaustive state of art with common clinical applications and to deliver future perspectives and methodological improvements of this specific topic.

The book published by Springer, containing more than 500 pages, a huge number of very high quality color illustrations, tables, and diagrams, is structured into almost 30 wellestablished chapters and an index.

After a very interesting extension of the historical origins and background of radioguided surgery as well as of physical principles of radiation detection, a significant description of techniques and potentials of the radioguided sentinel lymph node mapping on breast cancer is provided. Breast cancer and melanoma are certainly the malignancies in which radioguided surgery has shown its highest impact on life quality of our patients. The authors report interesting data on the impact of radioguided surgery on survival as well as trends for the future of sentinel lymph node mapping in the new era of personalized therapy. Thereafter, sentinel lymph node mapping and radioguided surgery of primitive cervical, thoracic, abdominal, and pelvic tumors are treated in an extensive way. This is asso-

Domenico Rubello

domenico.rubello@libero.it; rubello.domenico@azisanrovigo.it

1 Groupement Hospitalier Est, Bron, France

2 Biophysique - Faculté Charles Mérieux Lyon, Lyon, France

3 Santa Maria della Misericordia Hospital, Rovigo, Italy ciated with a wide-ranging list of references for each chapter, in order to incite the reader to deepen particular arguments of personal clinical interest. Moreover, an entire chapter is devoted to FDG-directed surgery and interventional procedures, a field in which several challenges are described. This topic has a significant impact given the widespread use of FDG-PET/CT in the last 15 years. At last, future directions of radioguided surgery using radioisotopes as well as new tracers, such as fluorescent or magnetic tracers, are thoroughly examined together with the description of initial experiences about fusion of SPECT with ultrasonography imaging. The last chapter of the book is dedicated to case-based issues and solutions from a theoretical and practical point of view. Thanks to the well-organized, didactic structure that involves the reader as protagonist in achieving the final judgment, this chapter can serve as a comprehensive tool not only for nuclear physicians but also for surgeons, as well as all members of the broader medical community who have a strong interest in this topic.

This guide is fluent and easy to read, simple to understand even when dealing with complex issues, and enriched by explanatory images and tables.

The final editorial outcome is a very comprehensive, clear, and well-illustrated set of high-quality, wide-ranging intellectual work that fulfills its objective to pave the way for the virtual connection between surgery and nuclear medicine in order to enhance the daily collaboration among physicians of different backgrounds and to offer the best standard of care to our patients.

In conclusion, this book represents a qualified, extensive, and updated text on the role of nuclear medicine in the field of radioguided surgery. Therefore, we highly recommend this volume, not only to experts but also to residents, clinicians, and to everyone who wants to be introduced to this emerging topic.

\section{Compliance with ethical standards}

Conflict of interest The authors have nothing to declare. 